\title{
Transition from multiple to single microcontact conduction during hot switching of microelectromechanical switches with ball-shaped dimples
}

\author{
Linda L. W. Chow and Steven A. Schrader \\ Department of Mechanical Engineering, University of Michigan, Ann Arbor, Michigan 48109 \\ Katsuo Kurabayashi ${ }^{\text {a) }}$ \\ Department of Mechanical Engineering, University of Michigan, Ann Arbor, Michigan 48109 \\ and Department of Electrical Engineering and Computer Science, University of Michigan, \\ Ann Arbor, Michigan 48109
}

(Received 27 April 2006; accepted 19 July 2006; published online 25 September 2006)

\begin{abstract}
Previous studies of electron transport within direct contact microelectromechanical switches have found that conduction occurs via nanoscale contact asperities. It has been claimed that reduced contact resistance can be achieved by using multiple contact switches; however, the ability of these switches to enhance power handling or lifetime remains a question. To study the contact mechanism, single-input-multiple-output switches with ball-shaped dimples were specially designed and tested. At all voltage levels of hot-switching operation, uneven current sharing among the outputs was observed. Furthermore, at softening voltage, an irreversible multiple to single conduction transition occurs and is found to alternate among different outputs. () 2006 American Institute of Physics. [DOI: $10.1063 / 1.2352041]$
\end{abstract}

Direct contact microelectromechanical system (MEMS) switches offer significant technological advantages over their solid-state counterparts. Their low power consumption, low insertion loss, high isolation, and linearity make them attractive alternatives to solid-state switches in military and commercial radar systems, satellites, and wireless communications systems. ${ }^{1}$ Because of their ultrawide bandwidth ( $\mathrm{dc}$ to $100 \mathrm{GHz}$ ), radio frequency (rf) MEMS switches are also promising building blocks for radio-on-chip development. ${ }^{2}$ MEMS switches are mostly operated in two modes: (1) coldswitching operation and (2) hot-switching operation. Cold switching refers to switch closure before applying voltage and voltage removal before switch opening, while hot switching refers to the switch actuation synchronized with the electrical switching. Hot switching is detrimental to contact reliability, but a proper understanding of the contact mechanism during hot switching has yet to be established.

MEMS switches in hot-switching operation suffer from long-term reliability issues such as unstable contact resistance and contact adhesion. These reliability issues make it challenging for achieving high power handling capabilities ${ }^{3}$ and long lifetimes ${ }^{4}$ with these switches. Our previous paper ${ }^{5}$ has demonstrated that contact resistance can be reduced by adjusting the contact voltage to the softening voltage $V_{\text {softening. }}$ In a clean environment, contact adhesion can be attributed to either localized Joule heating or excessive mechanical deformation of contact asperities under high contact force.

Switch designs with multiple contact dimples have been suggested to reduce contact resistance, ${ }^{6}$ thereby reducing insertion loss. Furthermore, in low-force operation, contact adhesion is mainly induced by the excessive localized Joule heating. As a result, switch power handling is bounded by the maximum current $I_{\max }$ before permanent contact adhesion occurs. It is a common belief that in a multiple contact switch, if the current load is distributed equally among the

\footnotetext{
${ }^{a)}$ Electronic mail: katsuo@umich.edu
}

contacts, increasing the number of contacts should increase the maximum current. However, there are no existing studies that prove this hypothesis.

In this letter, single-input-multiple-output MEMS switches were designed to study the contact mechanism of individual contacts during hot-switching operation at a contact voltage, $V_{C} \ll V_{\text {softening }}$ and at $V_{C} \sim V_{\text {softening. Since con- }}$ tact resistance has been shown to remain equal to its dc value at frequencies up to $20 \mathrm{THz},{ }^{7}$ our experiments were simplified by taking dc measurements.

Figure 1 shows an optical image of a single-inputmultiple-output (one-input-four-output) gold MEMS switch used for our study and its measurement setup. It is an electrostatically actuated switch with the actuation circuit separated from the contact current measurement circuit. To minimize localized Joule heating which could lead to contact softening, a resistor $R_{0}$ of $1.5 \mathrm{k} \Omega$ was connected in series with the contact voltage. The contact voltage is applied to the switch beam via multiple contacts, and each is connected to a common ground. Current output is measured by a separate

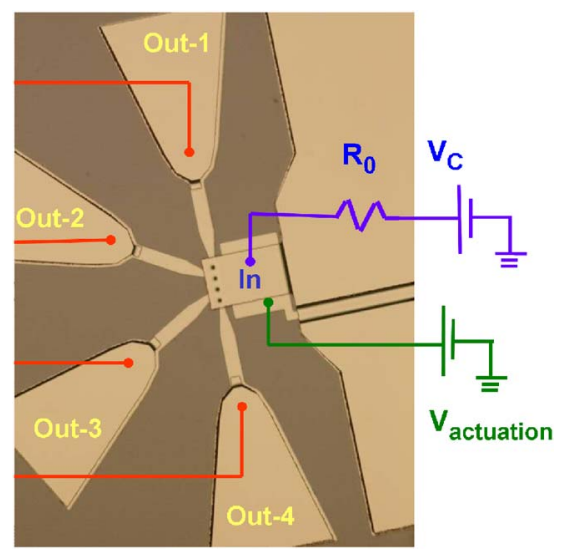

FIG. 1. (Color online) Optical image of a single-input-multiple-output switch with individual current measurement setup. 


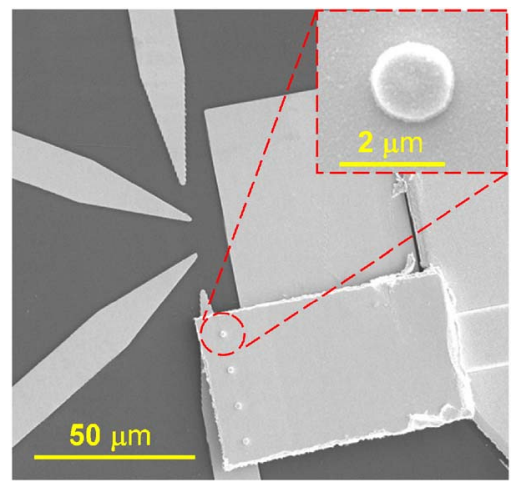

FIG. 2. (Color online) SEM images of the MEMS switch shown in Fig. 1 which has been flipped over to reveal the contact dimples.

ammeter (dc current mode in a 6.5 digit multimeter, Agilent 34401A).

To precisely characterize the contact mechanism, our switch was designed such that each output path was identical in layout design. The switch dimples are all ball shaped. This dimple shape results in a much smaller contact area than flat ones, which are normally found in standard MEMS switch designs. The switch presented typically yields a contact resistance on the order of $R_{C}=0.5 \Omega$. It follows that the diameter of the effective contact area is smaller than $68 \mathrm{~nm}$, which is given by Eq. (1) according to Wexler, ${ }^{8}$

$$
R_{C}=\gamma R_{M}+R_{S}=\frac{(1+1.83(\lambda / a))}{(1+1.33(\lambda / a))} \frac{\rho}{2 a}+\frac{4 \rho \lambda}{3 \pi a^{2}},
$$

where $\rho$ is the electrical resistivity (measured as 3.6 $\times 10^{-8} \Omega \mathrm{m}$ using on-chip van der Pauw structures), $a$ is the contact radius, $\lambda$ is the electron mean-free path $(38 \mathrm{~nm}$ at room temperature $\left.{ }^{9}\right), R_{S}$ is the Sharvin resistance owing to electron boundary scattering in small constrictions $R_{M}$ is the Maxwell spreading resistance owing to lattice scattering of electrons, and $\gamma$ is an interpolation function. ${ }^{10}$ This calculation suggests that the ball-shaped dimple may allow physical contact to be formed only via a few contact asperities, possibly approaching a single asperity contact condition. It is most probable that the nanoscale contact occurs at the top of the hemispheric surface of each contact dimple.

Figure 2 shows the ball-shaped dimple design under scanning electron microscopy (SEM) imaging after flipping over the switch cantilever beam. The dimples are $1.5 \mu \mathrm{m}$ in diameter and at a pitch of $10 \mu \mathrm{m}$. The switch can be built on any planar substrate. In this letter, the substrate was a silicon wafer with a $1-\mu \mathrm{m}$-thick thermally grown oxide layer for electrical insulation. The nanoscale contact features were batch fabricated without using e-beam lithography, as shown in Fig. 3. Initially, a $30 \mathrm{~nm}$ of chromium followed by $200 \mathrm{~nm}$ of gold was sputtered and lithographically patterned to form the actuation and output electrodes [Fig. 3(a)]. The first layer of photoresist was spin coated and patterned as the switch anchor, followed by hard baking at $160{ }^{\circ} \mathrm{C}$ for $10 \mathrm{~min}$. The second layer of photoresist was then spun on and patterned to form the switch dimples and openings for the anchor [Fig. 3(b)]. The ball-shaped dimple photoresist mold was formed with anchor openings by fine tuning the UV exposure time and the second photoresist thickness. Optical diffraction near the edges of small $(1.5 \mu \mathrm{m}$ in diameter) circular lithography mask patterns served to yield the photoresist mold shape under these conditions. To further smooth the edges, the pho-

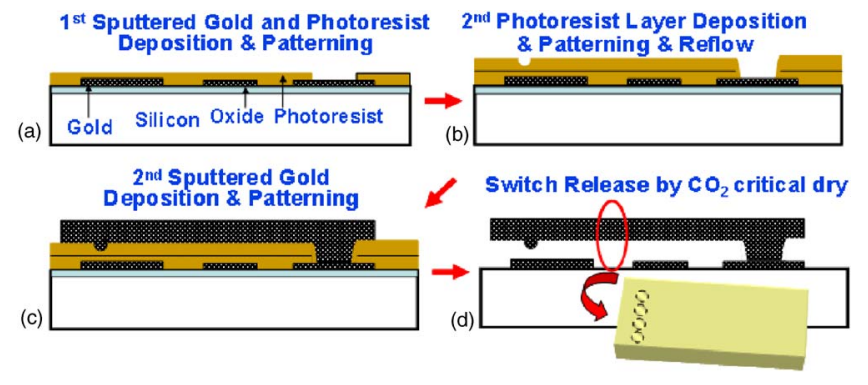

FIG. 3. (Color online) Fabrication process of a single-input-multiple-output switch with ball-shaped dimples.

toresist mold was hard baked and reflowed at $130{ }^{\circ} \mathrm{C}$ for $30 \mathrm{~min}$. To prevent scratching from debris generated during the die-saw process, a thick photoresist layer was blanket coated on the wafer without hard bake. The wafer was halfway cut to allow individual switch chips to be later separated from each other via fracture along cut lines. The protecting photoresist was removed using an acetone/isopropanol/deionized water rinse. Structural gold was then deposited via sputtering [Fig. 3(c)]. Individual switches were isolated by fracturing the wafer along the die-saw lines. Finally, the switches were released using a photoresist stripper followed by a $\mathrm{CO}_{2}$ critical point dry [Fig. 3(d)].

Prior to testing, a minimum actuation voltage of $95 \mathrm{~V}$ was recorded by slowly increasing the supplied voltage from zero until more than two contacts became electrically conductive, for theoretically, a free plane can be supported by three points without contact deformation. This voltage was used for the electrostatic actuation during all subsequent experiments. The contact force generated was estimated to be $<10 \mu \mathrm{N}$ using a static pure bending analysis of an EulerBernoulli beam. This force is small enough to prevent significant surface degradation due to mechanical impacts during the switching cycles. It is interesting to note that, among 56 switches measured, single-output conduction was always observed when the switches were first electrically closed. It was found that an increase in actuation voltage of approximately $5 \mathrm{~V}$ was required to achieve three- or four-output conduction.

Figure 4 shows the typical current evolution during hot

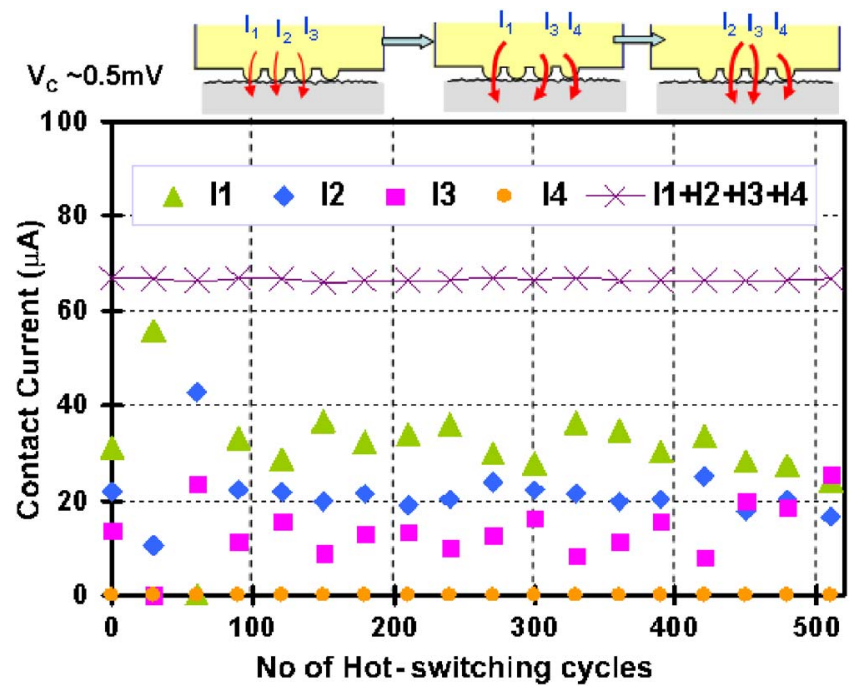

FIG. 4. (Color online) Individual current measurement from a single-inputfour-output switch during hot switching at low contact voltage. 


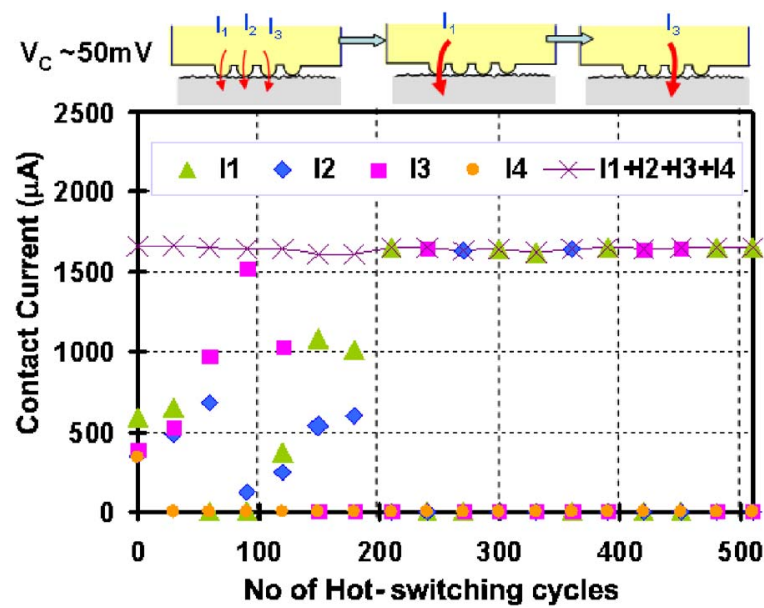

FIG. 5. (Color online) Individual current measurement at a single-inputfour-output switch in hot switching at high contact voltage, $V_{\text {softening. }}$.

switching for a low contact voltage $V_{C}$ of about $0.5 \mathrm{mV}$. This voltage is much smaller than $V_{\text {softening }}$ given by Eq. (2) according to Jensen et al.,

$$
V_{\text {softening }}=\sqrt{\frac{4 R_{C} L}{\gamma R_{M}}\left(T_{C}^{2}-T_{0}^{2}\right)},
$$

where $T_{C}$ and $T_{0}$ are the temperatures in kelvins of the contact asperities and the bulk of the switch dimple, respectively (ambient temperature $=20^{\circ} \mathrm{C}$ ). $T_{C}$ has been experimentally found to be $60-80{ }^{\circ} \mathrm{C}$. Hence, $V_{\text {softening }}$ is calculated to be $50-60 \mathrm{mV}$.

With a high contact voltage, $V_{C} \sim V_{\text {softening }}=50 \mathrm{mV}$, as in Fig. 5, the behavior at individual contacts varies dramatically. We note the following observations. (i) Only a portion of the contacts were conductive for a given cycle. (ii) The current load was unevenly distributed among the contacts, differing by several folds. (iii) Although individual output current varied, total current remained essentially constant during each hot-switching cycle. (iv) Multiple-output conduction became single output after several hundred hotswitching cycles. (v) After transitioning to single-output conduction, no further multiple-output conduction was observed. (vi) Single-output conduction can alternate between outputs during subsequent hot-switching cycles. Surprisingly, these findings indicate that the multiple dimple structure contributes little to power handling enhancement. The singlecontact conduction mode could make sudden contact adhesion more likely to occur during high power hot switching.
Our recent observation of failed switches using SEM suggests that nanowire can be drawn from the contact dimples during hot switching. We postulate that the observed transitional phenomenon may result from surface morphology evolution associated with different contact asperities being elongated to different degrees during the switching cycles. Further investigations of this mechanism are currently underway.

In conclusion, we have designed single-input-multipleoutput MEMS switches to study the contact mechanism during hot switching of multiple contact switches. To approach single asperity conduction, switch dimples were fabricated to be nanoscale and ball shaped. Throughout the entire range of contact voltages, the current load was found to be unevenly distributed among the contacts, differing by several folds. While maintaining the same equivalent contact resistance, the current through each output will vary slightly for low contact voltages and vary dramatically for contact voltages greater than or equal to the softening voltage. The transition from multiple- to single-output conduction is irreversible; however, the conduction can alternate between outputs during subsequent cycles. Contrary to the existing common belief, multiple contacts do not enhance power handling, but could extend the switch lifetime through slowing down the surface degradation via the alternating conduction mechanism. These findings provide insight into design and operation guidelines that will enable direct contact MEMS switches to achieve reliable hot switching.

This work is supported under the National Science Foundation with Grant No. ECS-0330963.

${ }^{1}$ G. M. Rebeiz, RF MEMS Theory, Design, and Technology (Wiley, Hoboken, NJ, 2003).

${ }^{2}$ P. Gammel, G. Fischer, and J. Bouchaud, Bell Labs Technical Journal 10, 29 (2005).

${ }^{3}$ Z. Wang, L. Chow, J. L. Volakis, K. Saitou, and K. Kurabayashi, Proceedings of the 2005 IEEE Antennas and Propagation Society International (IEEE, Piscataway, NJ, 2005), Vol. 1A, p. 81-84.

${ }^{4}$ J. Maciel, S. Majumder, R. Morrison, and J. Lampen, Proc. SPIE 5343, 9 (2003).

${ }^{5}$ B. D. Jensen, L. L. W. Chow, K. Huang, K. Saitou, J. L. Volakis, and K. Kurabayashi, J. Microelectromech. Syst. 14, 935 (2005).

${ }^{6}$ S. Majumder, N. E. McGruer, G. G. Adams, P. M. Zavracky, R. H. Morrison, and J. Krim, Sens. Actuators, A 93, 19 (2001).

${ }^{7}$ J. D. Lavers and R. S. Timsit, IEEE Trans. Compon. Packag. Technol. 25, 446 (2002)

${ }^{8}$ G. Wexler, Proc. Phys. Soc. London 89, 927 (1966).

${ }^{9}$ N. W. Ashcroft and N. D. Mermin, Solid State Physics, 1st ed. (Holt, Rinehart and Winston, New York, 1976).

${ }^{10}$ B. Nikolic and P. B. Allen, Phys. Rev. B 60, 3963 (1999).

${ }^{11}$ B. D. Jensen, K. Huang, L. L. W. Chow, and K. Kurabayashi, Appl. Phys. Lett. 86, 023507 (2005). 